\title{
Research of the Trajectory Planning of Performance Test Manipulator
}

\author{
1J. Liu \& C.X. Wu, ${ }^{2}$ J.Qu \& X.F. Wang \\ ${ }^{1}$ Technology Center of Oceanographic Instrumentation Shandong, Qingdao, China \\ ${ }^{2}$ Institute of Oceanographic Instrumentation Shandong Academy of Sciences, Qingdao, China
}

KEYWORD: manipulator; trajectory planning; cubic spline function

ABSTRACT: The trajectory of HY-3 manipulator is planned using the cubic spline function which is adopted for interpolation of the angle of manipulator's joint. From simulation experiments, conclusion was drawn that the planned trajectory is smooth and continuous in order to ensure the manipulator running steadily and meeting the requirements of tests.

\section{INTRODUCTION}

The door hinge durability test is the key part of reliability testing which plays an important role in the quality of home appliances. Conventional hinge life test machine were simple pneumatic equipment and its running track is fixed, unable to meet the test requirements of different types of products. HY-3 type appliance performance test manipulator is a new generation of test equipment and it is space joint structure, having a plurality of degrees of freedom. HY-3 manipulator can plan different trajectories, able to complete all kinds of complex tests. The manipulator appearance is shown in Fig1.

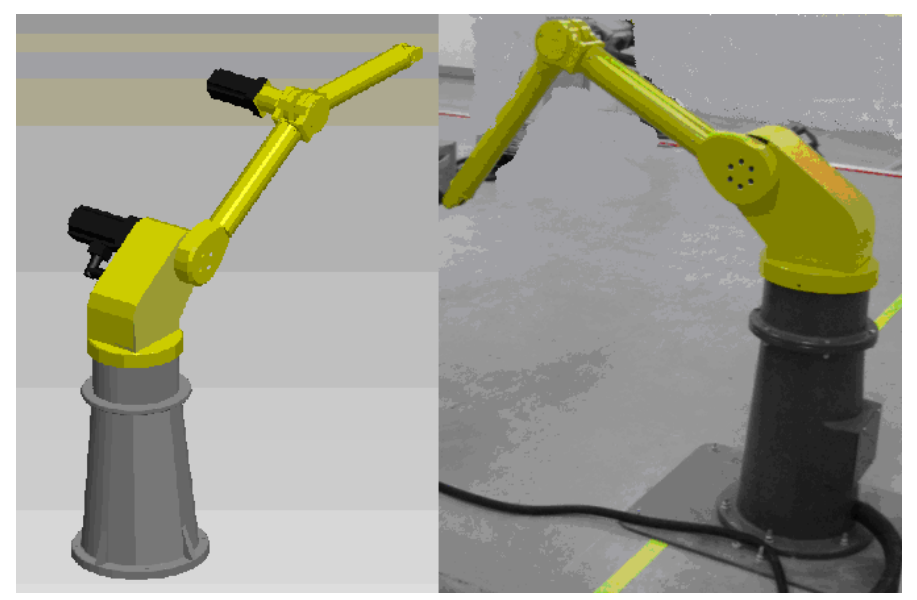

Figure 1. Outside drawing of HY-3 performance test manipulator.

Because the specifications styles and sizes of doors arevarious, we need to plan different trajectories for manipulator to meet different test requirements during the appliance door hinge life test. Manipulator's trajectory planning is to meet the requirements of the job tasks in order to calculate and set the path, orientation, speed and acceleration of end effector's position and posture changes artificially, during the operation. Manipulator's trajectory planning is a very important research. For multijoint manipulator, the planning is also a complex nonlinear problem.

\section{JOINT SPACE TRAJECTORY PLANNING}

The main task of trajectory planning of manipulator is to choose a reasonable polynomial function or linear function to execute interpolation operation. The main purpose is to smooth and stabilize the motion of manipulator and keep the motion of the joint within allowed range .This article discusses the trajectory planning and trajectory generation method of manipulator only in the joint space or Cartesian coordinate system. 
Domestic and foreign scholars have studied many trajectory planning of manipulator in nonlinear systems. Aspinwall proposed the pulse construction method. In this method, the motion control and vibration of manipulator was attributed to the non-linear two point boundary value for solution(Aspinwall D. M. 1980). Jankowski introduced inverse system method, using the state feedback and dynamic pre-compensation get to decouple linear systems(Jankowski K.P. 1993). However, these methods are complicated and there are certain difficulties to be applied to multi-DOF trajectory planning system. In this paper, we apply the cubic polynomial interpolation function into the studies of HY-3 type manipulator trajectory planning.

We use the node to represent the status of manipulator when carrying out the trajectory planning(He Yong. 2003). According to the working requirements, we set out its starting point, end point and an intermediate point of them called waypoints, when describing the movement of manipulator detailed. In working condition, the joint angle $\theta_{0}$ of the initial action position is known and the joint angle $\theta_{n}$ of the action target position can be obtained by inverse kinematics of space. So, there is a smooth interpolation function $\theta(t)$ which can be used to describe the relationship of the manipulator joint angle between the initial position and the target position in time interval $\left[t_{0}, t_{n}\right]$ (Xiong You-lun. 2001). The boundary conditions are:

$\theta\left(t_{0}\right)=\theta_{0} ; \theta\left(t_{n}\right)=\theta_{n}$

In the course of work, the movement of manipulator should be smooth. The chosen trajectory describing function must be continuous because unsmooth movement will lead vibration and shock and exacerbate wear of mechanical parts. In addition to satisfy the boundary conditions, the first derivative (velocity) of the interpolation function and sometimes the second derivative (acceleration) should be continuous(Yan Xue-gao. 1992).

To achieve smooth motion, the trajectory function of each joint must meet at least four constraints. In addition to location constraints, there are speed constraints:

$\dot{\theta}\left(t_{0}\right)=0 ; \dot{\theta}\left(t_{n}\right)=0$

With four constraints given above, a cubic polynomial can be uniquely identified to use to describe the movement of the manipulator joint:

$\theta(t)=\alpha_{0}+\alpha_{1} t+\alpha_{2} t^{2}+\alpha_{3} t^{3}$

Solving equations by the boundary conditions, we can get $\alpha_{0} 、 \alpha_{1} 、 \alpha_{2} 、 \alpha_{3}$. Substituting the parameters into equation (3), we can get the cubic polynomial interpolation function which can meet requirements of continuous smooth motion to make sure that the articulation speed of the start position and end position are 0 .

$\theta(t)=\theta_{0}+\frac{3}{t_{n}^{2}}\left(\theta_{n}-\theta_{0}\right) t^{2}-\frac{2}{t_{n}^{3}}\left(\theta_{n}-\theta_{0}\right) t^{3}$

The expressions of joint angular velocity and angular acceleration are:

$\dot{\theta}(t)=\frac{6}{t_{n}^{2}}\left(\theta_{n}-\theta_{0}\right) t-\frac{6}{t_{n}^{3}}\left(\theta_{n}-\theta_{0}\right) t^{2}$

$\ddot{\theta}(t)=\frac{6}{t_{n}^{2}}\left(\theta_{n}-\theta_{0}\right)-\frac{12}{t_{n}^{3}}\left(\theta_{n}-\theta_{0}\right) t$

In the case of articulation speed of start point and end point is not 0 , the method is consistent with the aforementioned of determining cubic polynomial coefficients, just changing the speed constraints. To promote this method, we can see two adjacent path points on each joint as the start point and end point, and then determine the corresponding cubic polynomial interpolation function, connecting the path points smoothing. The cubic polynomial interpolation function can describe the trajectory between start and end points with any given position and velocity constraints. 


\section{THE APPLICATION OF CUBIC INTERPOLATION FUNCTION IN MANIPULATOR}

TRAJECTORY PLANNING

The appliance door performance test actually uses manipulator to imitate human hand to test door and hinge's strength and durability. According to the actual work situation of HY-3 appliance performance test manipulator, we substituted the known data into the formula (4)-(6) in order to obtain the angular displacement, angular velocity and angular acceleration function of different joint variables.

The curves are shown in figure 2-4 using MATLAB software(Yang Ming-sheng. 2003). Joint 1 is the front-end elbow of manipulator which drive forearm doing pitching motion. Joint 2 is the shoulder of manipulator which drive arm and forearm doing pitching motion. Joint 3 is the waist of manipulator which drive all parts of manipulator except the base doing rotational movement.
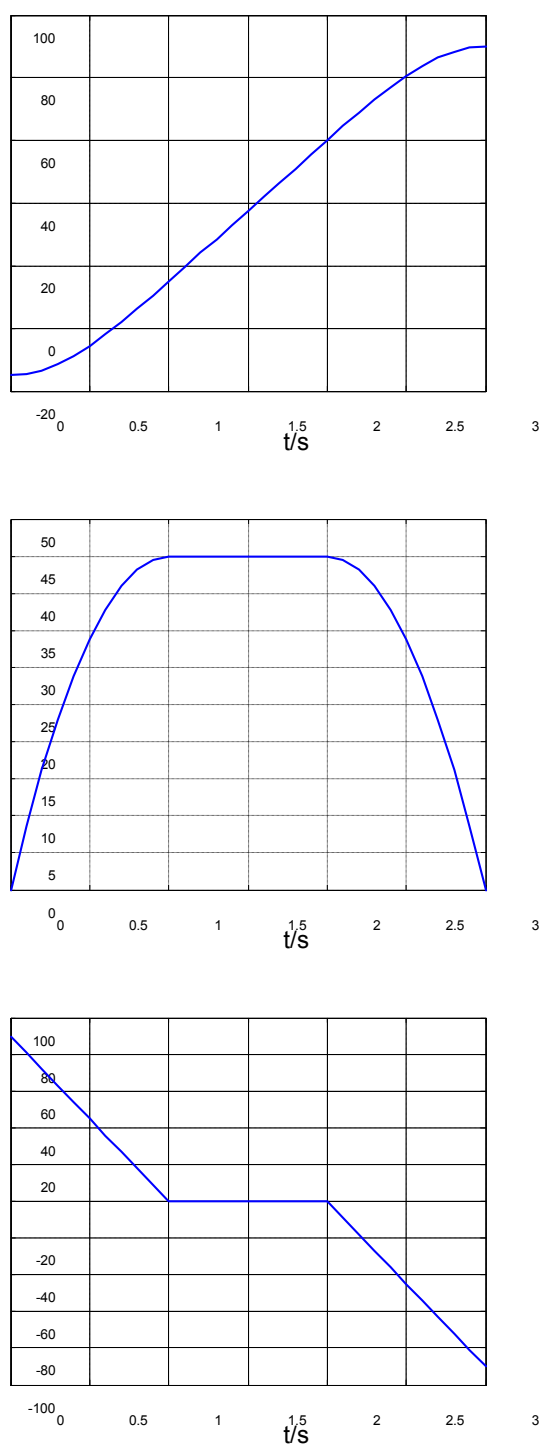

Figure 2. Chart of displacement, velocity and acceleration of joint 1. 

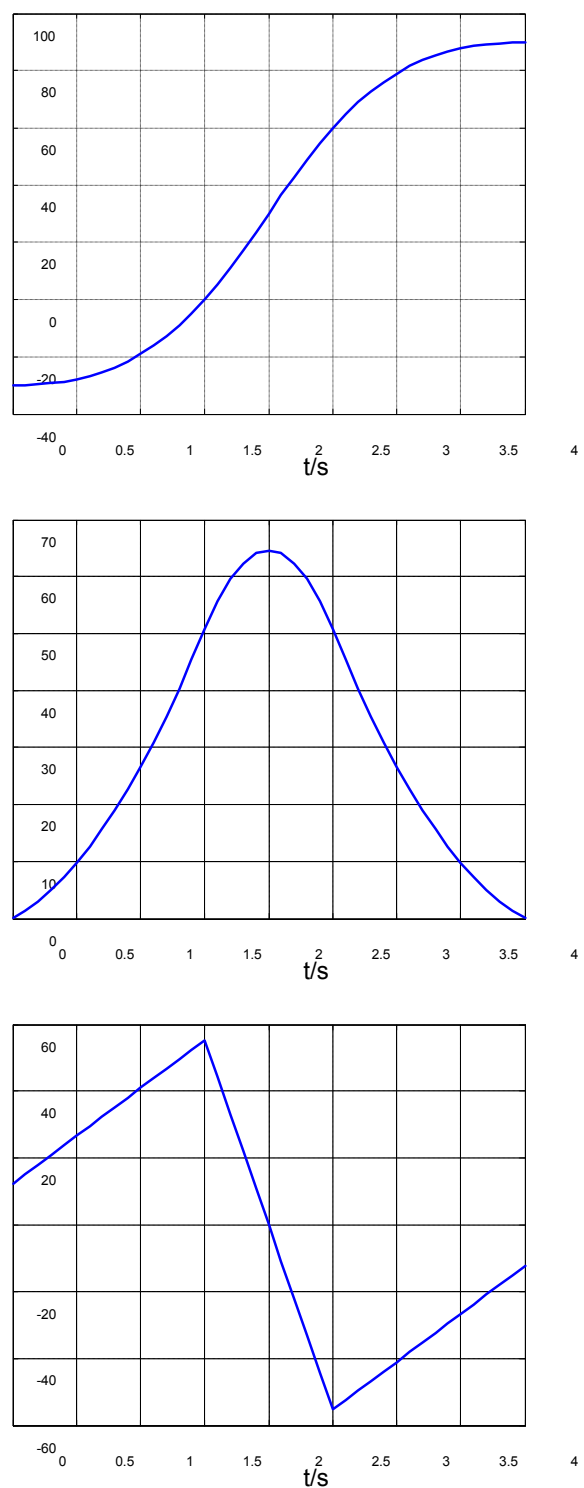

Figure 3. Chart of displacement, velocity and acceleration of joint 2. 

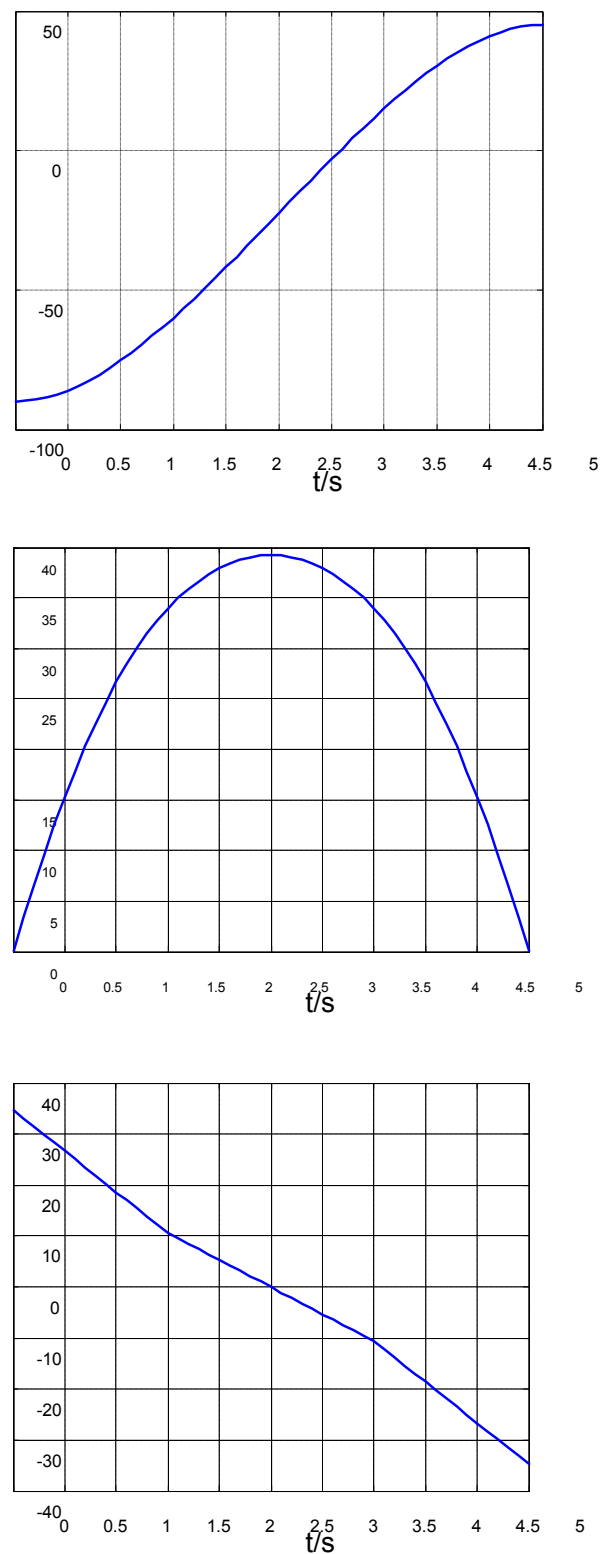

Figure 4. Chart of displacement, velocity and acceleration of joint 3.

The curves illustrate that the manipulator's displacement curve is smooth and the velocity and acceleration curve continuous. That means the manipulator is running smoothly in the work process except at the start and end point and the parts did not produce vibrations that ensure the door switch test smooth and continuous. Besides, with the extension of the set time, the angular velocity and angular will change more and more slowly. Therefore, under the permission of the test requirements and efficiency, operators should as far as possible to extend the set time. This help further reduce vibration and wear to increase the service life of the manipulator.

\section{CONCLUSIONS}

In this article, we do the trajectory planning using cubic polynomial interpolation function for HY-3 type manipulator which was brought in the household door switch test. As the result of actual test, the manipulator moves stablely and its trajectory is smooth which fully able to meet the test re- 
quirements. This method has very high practical value for manipulator trajectory planning which has small amount of calculation and is easy to be grasped.

\section{REFERENCES}

[1] Aspinwall D M. Acceleration Profiles for Minimizing Residual Response. Journal of Dynamic Systems,Measurement, and Control. 102,pp.3-6, 1980.

[2] He Yong \& Shen Qiong. The Position Control of Prosethema Manipulator with Five Degree of Freedom. Journal of Donghua University, 29(1),pp.45-51, 2003.

[3] Jankowski K P. Inverse Dynamics Task Control of Flexible Joint Manipulators-Part I: Continuoutime Approach, Part II: Discrete-time Approach. Mechanism and Machine Theory, 28(6),pp.741-749,1993.

[4] Xiong You-lun. Fundamentals of Robotics. Wuhan: Huazhong University of Science and Technology Press, 2001.

[5] Yan Xue-gao \& Meng Zheng-da. Robot Principle. Nanjing: Southeast University Press, 1992.

[6] Yang Ming-sheng, Xiong Xi-wen \& Lin Jian-hua. MATLAB Basis and Mathematical Software. Dalian: Dalian University of Technology Press, 2003. 